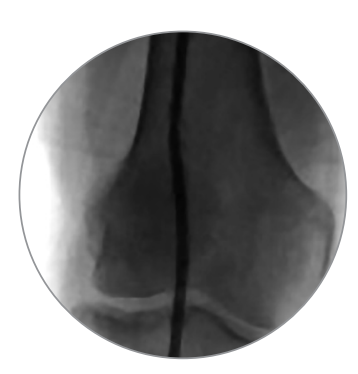

\section{Revascularización de arteria poplítea con arteria ciática persistente bilateral: presentación de un caso}

\author{
Popliteal Revascularization in Bilateral Persistent Sciatic Artery: \\ Case Report
}

Fernando José Meléndez Negrette José Miguel Hidalgo ${ }^{2}$ Sergio Álvarez ${ }^{2}$ Emilio Sanín ${ }^{2}$

Palabras clave (DeCS)

Ciática

Arteria ilaca

Extremidades

Isquemia

\section{Key words (MeSH)}

Sciatic

Iliac artery

Extremities

Ischemia

'Radiólogo, fellow de Radiología Intervencionista, Universidad de Antioquia. Medellín, Colombia.

${ }^{2}$ Radiólogo, especialista en Radiología Intervencionista, Departamento de Intervencionismo, Hospital Pablo Tobón Uribe. Medellín, Colombia.
Mujer de 81 años con antecedente de dislipidemia, hipertensión, enfermedad coronaria y tabaquismo, con cuadro clínico de 15 días de evolución consistente en dolor en miembro inferior derecho. Al examen físico se evidenció retraso en el llenado capilar de más de 2 segundos y necrosis del primer dedo. Se realizó una pletismografía que demostró compromiso infrapoplíteo grave, por lo cual cirugía vascular solicitó arteriografía e intervención según hallazgos, ya que por sus comorbilidades, la paciente no era candidata a manejo quirúrgico.

Se le realizó arteriografía por punción descendente desde la arteria femoral común derecha; como no se pudo canular con introductor vascular corto $5 \mathrm{Fr}$ la arteria femoral superficial, se decidió inyectar para arteriografía (figura 1a), la cual evidenció marcada hipoplasia de la arteria femoral superficial sin conexión con la arteria poplítea en el canal de los aductores (figura 1b). Por lo anterior, se decidió realizar punción ascendente con aguja $21 \mathrm{G}$ desde la arteria tibial posterior y se posicionó un dilatador 5 Fr desde donde se avanzó una guía 0.018 ", la cual ascendió hacia la zona poplítea y, tras múltiples intentos, se dirigió hacia un trayecto lateral (figura 2). Entonces, se decidió realizar un aortograma y arteriografía pélvica, examen que demostró arterias ciáticas persistentes de forma bilateral (figura 3), sin aneurismas y oclusión de la arteria poplítea derecha

\section{Presentación del caso}

(figura 4). Se practicó angioplastia mediante abordaje contralateral con balón sobre guía, con recanalización satisfactoria de la arteria poplítea, descendiendo por la arteria ciática persistente (ACP) (figura 5a), y con mejoría de la perfusión distal del pie (figura 5 b).

\section{Discusión}

La arteria ciática persistente (ACP) es una anomalía vascular muy rara, resultado de la persistencia de una arteria que normalmente regresa en los primeros 3 meses de la gestación. Durante las primeras semanas esta arteria aporta la irrigación de los miembros inferiores $\mathrm{y}$, posteriormente, cuando el embrión mide $12 \mathrm{~mm}$ la arteria femoral superficial incrementa de tamaño, se anastomosa con la arteria ilíaca externa y posteriormente se atrofia la arteria ciática para convertirse en las ramas distales de la arteria glútea inferior, superior y del nervio ciático. Su incidencia es del $0,025 \%$ al $0,04 \%$ y la bilateralidad solamente se encuentra en el $12-30 \%$ de los casos (1). Es derecha en el $50 \%$ de los casos, izquierda en el $20 \%$ y bilateral en menos del $30 \%$. Se presenta en ambos sexos por igual. La gran mayoría de los casos son asintomáticos y los síntomas suelen ocurrir a la edad de 40-50 años, con claudicación, aneurisma y raramente con ciática $(2,3)$. Esta anomalía se ha asociado a alteraciones mullerianas, fístulas arteriovenosas, hipertrofia de la extremidad y arteria subclavia derecha retroesofágica (4). 

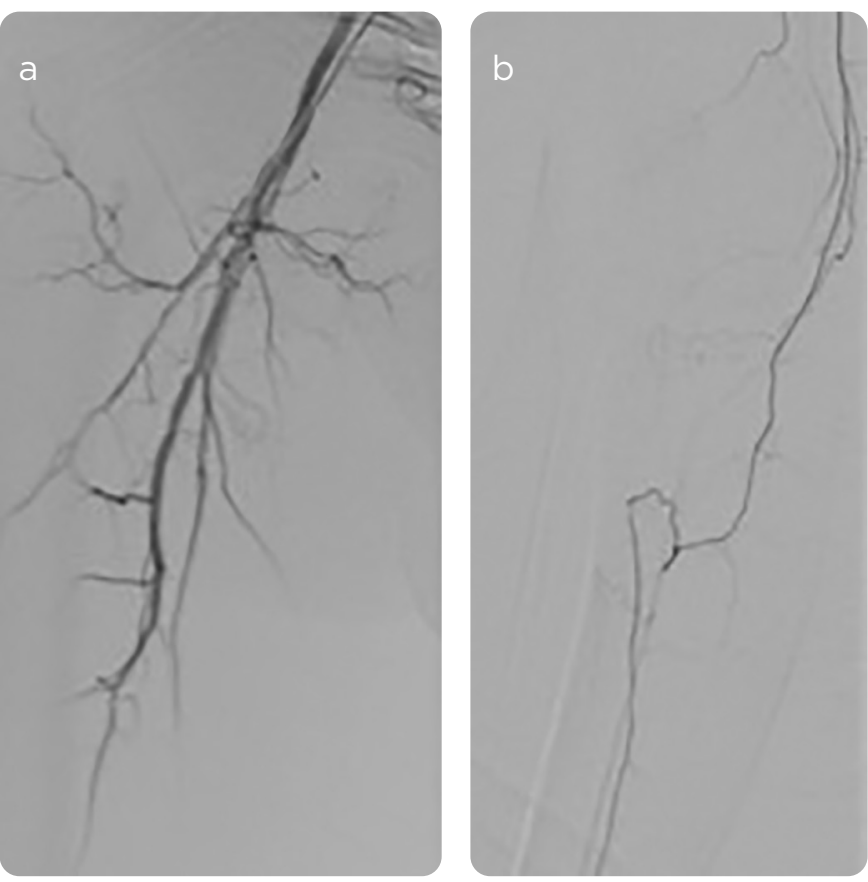

Figura 1. Arteriografía. a) hipoplasia de la arteria femoral superficial. b) colateral medial de arteria femoral superficial que se anastomosa con arteria tibial posterior.

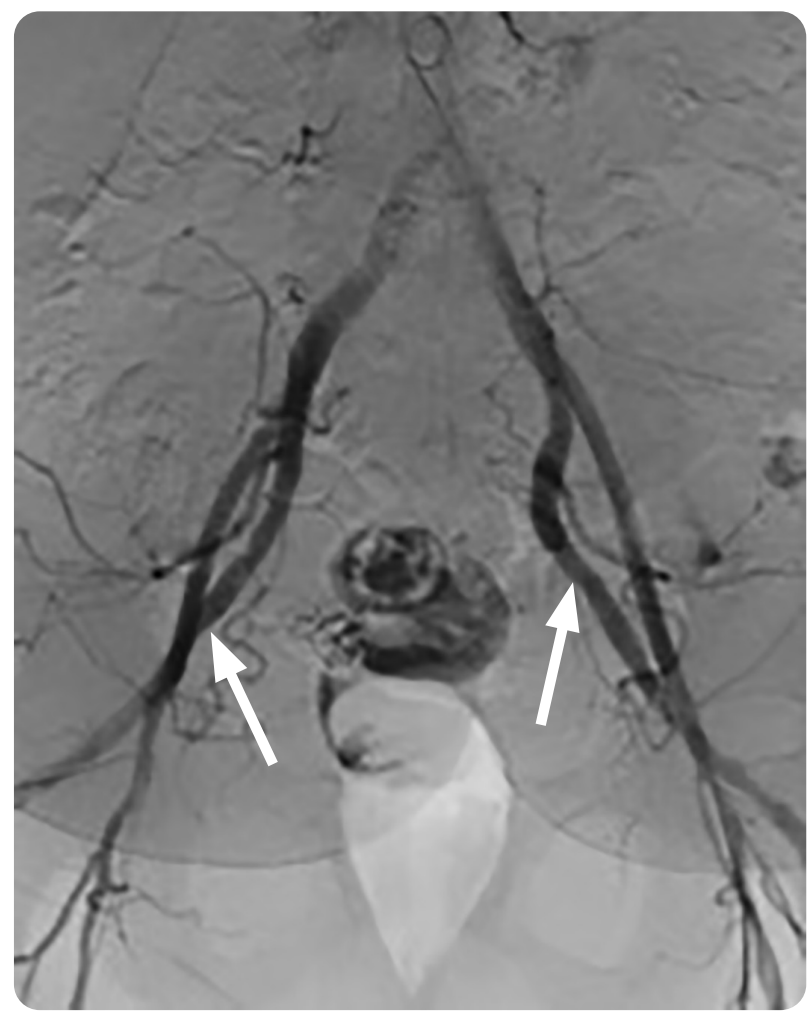

Figura 3. Arteriografía pélvica: arteria ciática persistente bilateral como continuidad de las arterias ilíacas internas (flechas).

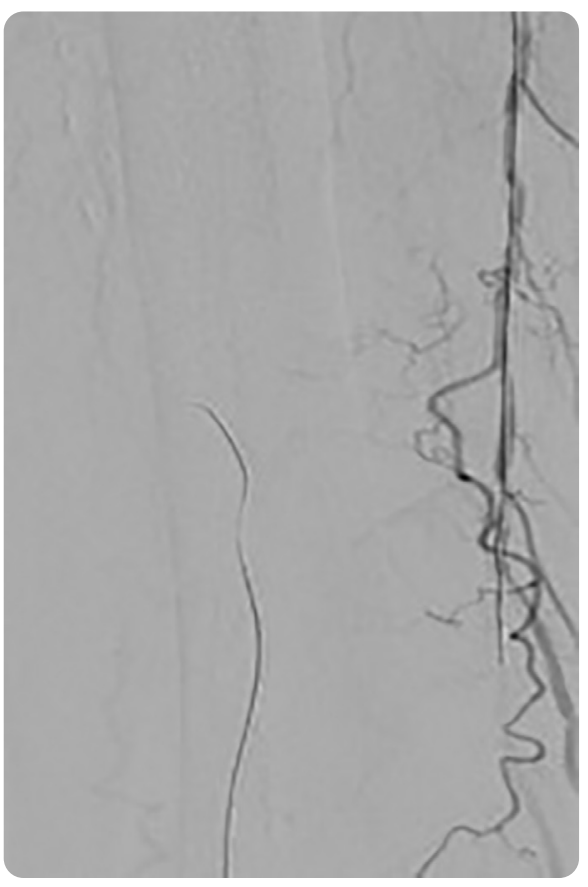

Figura 2. La guía por punción ascendente desde la arteria tibial posterior tiene un trayecto lateral en contraste con el borde medial de la arteria femoral superficial hipoplásica.

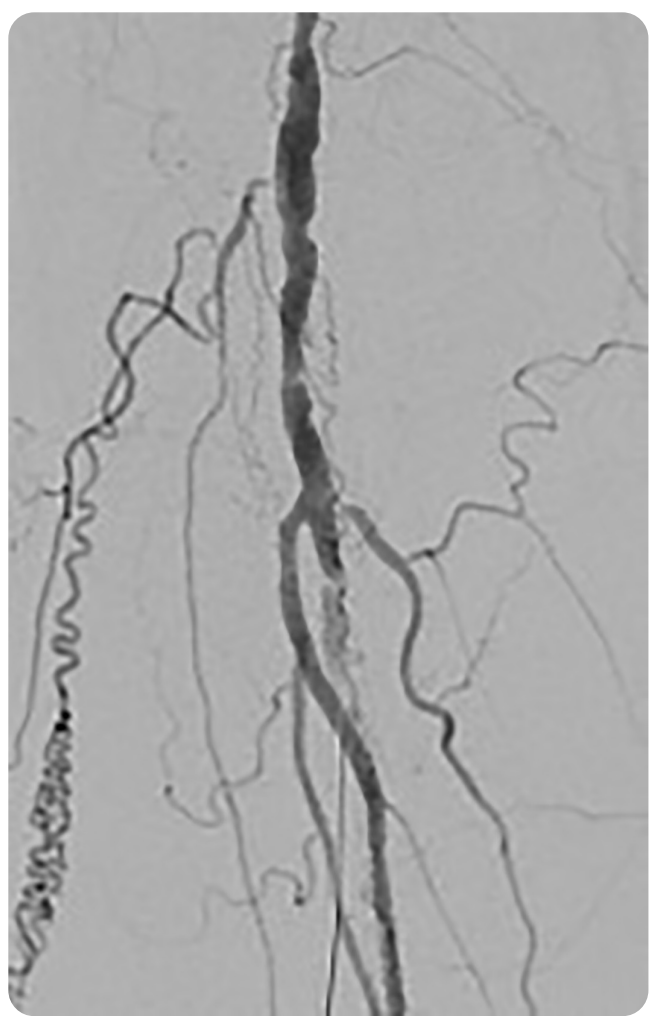

Figura 4. Enfermedad ateromatosa de la arteria con oclusión de la arteria poplítea con colaterales. 

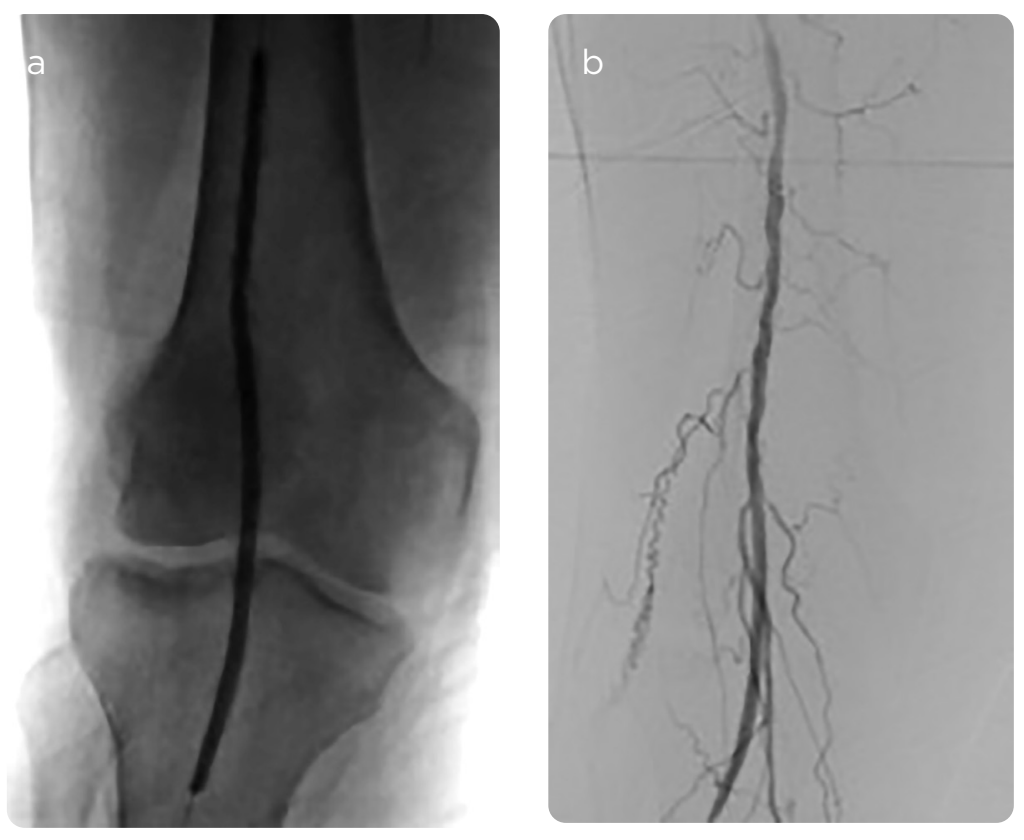

Figura 5. a) Angioplastia con balón convencional del segmento ocluido. b) Repermeabilidad de la arteria poplítea de forma satisfactoria.

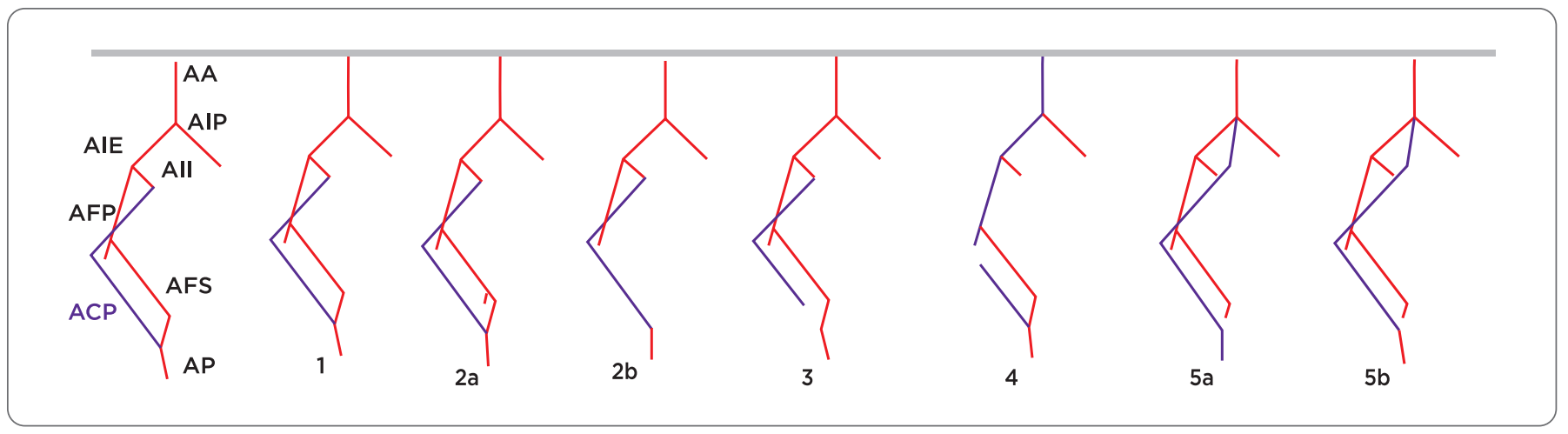

Figura 6. Diagrama de clasificación de la arteria ciática persistente de acuerdo con la persistencia, hipoplasia o agenesia.

Algunos autores sugieren que si la arteria femoral es hipoplásica se favorece la persistencia de la arteria ciática (4).

Anatómicamente, la arteria es prolongación de la arteria ilíaca interna y se continúa por el foramen ciático a través del músculo piriforme acompañando el nervio ciático, para luego descender por el miembro inferior medial al nervio y prosiguiendo con la arteria poplítea (5).

Green describió esta anomalía en 1832, en estudios cadavéricos, y se realizó una clasificación dependiendo de la persistencia, hipoplasia o agenesia de la arteria ciática y femoral superficial: El tipo 1: una ACP completa, junto con una arteria femoral normal; el tipo 2: una ACP completa con desarrollo femoral incompleto; en el tipo 2a, la arteria femoral está presente, pero se estrecha y no llega a la arteria poplítea, como en el caso aquí descrito; en el tipo 2b, la arteria femoral está completamente ausente, en el tipo 3: una ACP incompleta en la que solo persiste el segmento proximal de la arteria ciática y las arterias femorales están completamente desarrolladas. El tipo 4: una ACP incompleta, en la que solo persiste el segmento distal de la arteria y las arterias femorales están completamente desarrolladas. El tipo 5: la ACP se origina en la arteria sacra mediana; el tipo 5a tiene una arteria femoral desarrollada y el tipo 5b tiene una arteria femoral poco desarrollada (figura 6) (4).

Al examen físico se puede identificar el signo de Cowie, la ausencia de pulso femoral, pero con pulso poplíteo patognomónico, aunque su ausencia no descarta la ACP (6). El aneurisma es la presentación más frecuente de la ACP, descrita hasta el $42 \%$ de los casos, se detecta como una masa pulsátil en los glúteos producto del cizallamiento del paso de la arteria por el músculo piriforme y el ligamento sacroespinoso. Ocasionalmente crece y produce síntomas compresivos (7). Los autores están a favor de tratarlo, ya que se asocia a ruptura y embolismo (8). El segundo síntoma más frecuente es el embolismo (9).

La ACP se puede diagnosticar por Doppler, angiotomografía o angiografía por resonancia. Es importante el reconocimiento de esta variante, ya que en algunos casos se debería evitar un puente femoropoplíteo considerando que la arteria femoral esté ocluida (10).

Algunos autores están a favor de realizan estudios no invasivos en primera instancia, que permiten visualizar las relaciones anatómicas con el foramen ciático y valorar la existencia de aneurismas o trom- 
bosis, pues en la arteriografía se puede confundir el diagnóstico con oclusiones arteriales (5).

No está indicado el tratamiento en pacientes asintomáticos, pero sí se recomienda el seguimiento anual con Doppler para vigilar la formación de aneurismas (3). El tratamiento depende de los síntomas y la clasificación. En pacientes con embolismo, el uso de anticoagulación oral y análogos de prostaglandinas mejoran los síntomas de claudicación (11). Si se opta por el tratamiento médico, es necesario hacer seguimiento con Doppler e índice tobillo/brazo cada 3-6 meses.

En caso de aneurismas, se indica el tratamiento endovascular mediante embolización o colocación de stents recubiertos, o cirugía mediante bypass o aneurismectomía con interposición de colgajo venoso o prótesis, dependiendo de si la ACP es completa o incompleta, ya que una forma completa contraindica la oclusión de este vaso (8). En este caso fue pertinente el reconocimiento de dicha anomalía con el fin de encontrar el acceso que permitiera la recanalización vascular de forma satisfactoria. Llama la atención el no encontrar aneurisma y la causa ateroesclerótica de la oclusión poplítea.

\section{Referencias}

1. Mazet N, Soulier-Guerin K, Ruivard M, Garcier JM, Boyer L. Bilateral persistent sciatic artery aneurysm discovered by atypical sciatica: a case report. Cardiovasc Intervent Radiol. 2006;29(6):1107-10.

2. Wang B, Liu Z, Shen L. Bilateral persistent sciatic arteries complicated with chronic lower limb ischemia. Int J Surg Case Rep. 2011;2(8):309-12. doi: 10.1016/j. ijscr.2011.07.010.

3. Patel MV, Patel NH, Schneider JR, Kim S, Verta MJ. Persistent sciatic artery presenting with limb ischemia. J Vasc Surg. 2013;57(1):225-9. doi: 10.1016/j.jvs.2012.06.108.

4. Van Hooft IM, Zeebregts CJ, van Sterkenburg SM, de Vries WR, Reijnen MM. The persistent sciatic artery. Eur J Vasc Endovasc Surg. 2009;37(5):585-91. doi: 10.1016/j. ejvs.2009.01.014.

5. Jung AY, Lee W, Chung JW, et al. Role of computed tomographic angiography in the detection and comprehensive evaluation of persistent sciatic artery. J Vasc Surg. 2005;42(4):678-83.

6. Knight BC, Tait WF. Massive aneurysm in a persistent sciatic artery. Ann Vasc Surg. 2010;24(8):1135.e13- 1135.e18. doi: 10.1016/j.avsg.2010.05.017.

7. Wijeyaratne SM, Wijewardene N. Endovascular stenting of a persistent sciatic artery aneurysm via retrograde popliteal approach: a durable option. Eur J Vasc Endovasc Surg. 2009;38(1):91-2. doi: 10.1016/j.ejvs.2009.03.007.

8. Mascarenhas de Oliveira F, de Souza Mourão G. Endovascular repair of symptomatic sciatic artery aneurysm. Vasc Endovascular Surg. 2011;45(2):165-9. doi: $10.1177 / 1538574410389340$

9. Yamamoto H, Yamamoto F, Ishibashi K, et al. Intermediate and long-term outcomes after treating symptomatic persistent sciatic artery using different techniques. Ann Vasc Surg. 2011;25(6):837.e9- 837.e15. doi: 10.1016/j.avsg.2011.02.017.

10. Brantley SK, Rigdon EE, Raju S. Persistent sciatic artery: embryology, pathology, and treatment. J Vasc Surg. 1993;18(2):242-8.

11. Kritsch D, Hutter HP, Hirschl M, Katzenschlager R. Persistent sciatic artery: an uncommon cause of intermittent claudication. Int Angiol. 2006;25(3):327-9.

\section{Correspondencia}

Fernando José Meléndez Negrette

Departamento de Radiología

Universidad de Antioquia

Medellín, Colombia

fernandomelendezun@gmail.com

Recibido para evaluación: 11 de abril de 2019

Aceptado para publicación: 1 de diciembre de 2019 